\title{
Comparison of Healthcare Costs Between Rheumatoid Arthritis Patients Treated with Infused Biologics After Switching from Another Biologic
}

\author{
Stephen S. Johnston • Donna McMorrow • \\ Amanda M. Farr $\cdot$ Paul Juneau $\cdot$ Sarika Ogale
}

Published online: 18 February 2015

(c) The Author(s) 2015. This article is published with open access at Springerlink.com

\begin{abstract}
Introduction While there is a substantial body of literature on the comparative healthcare costs of biologics used to treat rheumatoid arthritis (RA), nearly all of these investigations have been exclusively focused on anti-tumor necrosis factor- $\alpha$ (anti-TNF) agents in the setting of firstline biologic treatment. This study compared healthcare costs between RA patients treated with infused biologics after previously using at least one other biologic agent.

Methods Using a large US administrative claims dataset, adult RA patients initiating an infused biologic (abatacept, infliximab, tocilizumab) between January 1, 2010 and January 1, 2012 (initiation $=$ index) were identified.
\end{abstract}

\footnotetext{
S. S. Johnston $(\bowtie) \cdot$ D. McMorrow · A. M. Farr · P. Juneau Truven Health Analytics, 7700 Old Georgetown Rd, Ste 650, Bethesda, MD 20814, USA

e-mail: stephen.johnston@truvenhealth.com

D. McMorrow

e-mail: donna.mcmorrow@ truvenhealth.com

A. M. Farr

e-mail: amanda.farr@truvenhealth.com

P. Juneau

e-mail: paul.juneau@truvenhealth.com

S. Ogale

Genentech, Inc, 1 DNA Way, South San Francisco, CA 94080, USA

e-mail: ogale.sarika@gene.com
}

Rituximab was excluded because of unique dosing intervals, which make it difficult to determine treatment discontinuation using a claims database. Patients were required to have used one or more other biologic (infused or injected) at any time before index. Patients could contribute multiple observations to the dataset; one for each infused biologic they initiated between January 1, 2010 and January 1, 2012. A 6-month period before index was used to measure patient characteristics. A variable-length follow-up period after index was used to measure per-patient per-month (PPPM) healthcare costs, including biologic costs, RA-related healthcare costs, and all-cause healthcare costs. Generalized estimating equations models compared healthcare costs between the biologic agents, adjusting for patients' demographics and clinical characteristics.

Results The sample comprised 3,771 infused biologic initiations (abatacept $=1,759$; infliximab $=922$; tocilizumab $=1,090$ ); the mean age of participants was 55 years, $82 \%$ were female, and the median follow-up ranged from 251 to 280 days. Compared with other patients, patients treated with tocilizumab had significantly lower (all $P<0.05$ ) PPPM biologic costs (abatacept $=\$ 2,597$, infliximab $=\$ 3,141$, tocilizumab $=\$ 1,894)$, RA-related healthcare costs $\quad$ (abatacept $=\$ 2,929, \quad$ infliximab $=$ $\$ 3,598$, tocilizumab $=\$ 2,236$ ), and all-cause healthcare costs (abatacept $=\$ 3,735$, infliximab $=\$ 4,600$, tocilizum$\mathrm{ab}=\$ 3,042)$.

Conclusions Among RA patients treated with infused biologics after previously using at least one other biologic, patients treated with tocilizumab had the lowest real-world healthcare costs, largely driven by lower costs directly related to biologic treatment. Such biologic-related cost differences may be driven by variations in real-world treatment patterns (e.g., dose, escalation, treatment frequency). 


\section{Key Points}

While there is a substantial body of literature on the comparative healthcare costs of biologics used to treat rheumatoid arthritis (RA), nearly all of these investigations have been exclusively focused on antitumor necrosis factor- $\alpha$ (anti-TNF) agents in the setting of first-line biologic treatment.

This study compared healthcare costs between RA patients treated with infused biologics after previously using at least one other biologic agent.

Using a large US administrative claims dataset, adult RA patients initiating an infused biologic (abatacept, infliximab, tocilizumab) between January 1, 2010 and January 1, 2012 (initiation $=$ index) were identified.

A variable-length follow-up period after index was used to measure per-patient per-month healthcare costs, including biologic costs, RA-related healthcare costs, and all-cause healthcare costs.

Among RA patients treated with infused biologics after previously using at least one other biologic, patients treated with tocilizumab had the lowest realworld healthcare costs.

\section{Introduction}

In the treatment of rheumatoid arthritis (RA), biologic agents are recommended for patients who have experienced an inadequate response to conventional disease modifying anti-rheumatic drugs [1]. Among the biologic agents currently approved for treatment of RA, seven may be self-administered through subcutaneous injection (abatacept, adalimumab, anakinra, certolizumab, etanercept, golimumab, and tocilizumab) and five have to be administered through intravenous infusion by a healthcare provider (abatacept, golimumab, infliximab, tocilizumab, and rituximab). The choice between self-administered and healthcare provider-administered agents can depend on a number of factors, including patient and provider preferences, reimbursement, and insurance coverage policies [2-4].

The American College of Rheumatology recommends the use of anti-tumor necrosis factor- $\alpha$ (anti-TNF) therapies as the first-line option when initiating a patient with RA on biologic therapy [1]. Thus, most infused biologics, three of which are not anti-TNF therapies, are usually used in patients who have previously used at least one other biologic agent. Among such patients for whom a biologic with an infused route of administration has been chosen, the available therapies differ widely with respect to mechanism of action, frequency of administration, and variation in dosing options [5-8].

US payers are increasingly relying on real-world, comparative healthcare utilization and cost data when making decisions related to the coverage of therapies $[9,10]$. While there is a substantial body of literature on the comparative healthcare costs of biologics used to treat RA, nearly all of these investigations have been exclusively focused on antiTNF agents in the setting of first-line biologic treatment [11-19]. Though two prior studies have examined healthcare costs among patients treated with the infused biologics abatacept, infliximab, and rituximab, both were intended to be purely descriptive and made no attempts to adjust for inherent differences in patient characteristics that may drive differences in healthcare costs [20, 21]. Furthermore, to date, no prior studies have examined the costs of RA patients treated with tocilizumab. Thus, the objective of this retrospective, observational cohort study was to compare healthcare costs between RA patients treated with infused biologics after previously using at least one other biologic agent. This study focuses specifically on infused biologics to provide specific, comparative information on these agents to inform treatment choice when circumstances such as insurance coverage or patient/provider preferences necessitate the use of this route of administration.

\section{Methods}

\section{Data and Setting}

This was a retrospective, observational cohort study based on US administrative claims data extracted from the Truven Health MarketScan ${ }^{\circledR}$ Commercial Claims and Encounters (Commercial) and Medicare Supplemental and Coordination of Benefits (Medicare Supplemental) databases. These databases comprise enrollment information, demographic information, and inpatient medical, outpatient medical, and outpatient pharmacy claims data collected from over 300 large self-insured US employers and over 25 US health plans. The Commercial database includes information on individuals who are under the age of 65 and are the primary insured or a spouse or dependent thereof. The Medicare Supplemental database includes information for individuals who are Medicare eligible and have a supplemental insurance paid for by their current or former employer. The study databases contained data for over 40 million unique individuals in 2011. These 
databases have been used in multiple studies related to RA [22].

The study databases satisfy the conditions set forth in Sections 164.514 (a)-(b)1ii of the Health Insurance Portability and Accountability Act of 1996 privacy rule regarding the determination and documentation of statistically de-identified data. Because this study used only de-identified patient records and does not involve the collection, use, or transmittal of individually identifiable data, Institutional Review Board approval to conduct this study was not necessary.

As described in greater detail below, study variables were measured from the database using enrollment records, International Classification of Diseases, 9th Revision, Clinical Modification (ICD-9-CM) codes, Current Procedural Technology, 4 th edition $\left(\mathrm{CPT}-4^{\circledR}\right)$ codes, Healthcare Common Procedure Coding System (HCPCS) codes, and National Drug Codes (NDCs), as appropriate [23].

\section{Patient Selection Criteria}

Patients were included in the analysis if they met all of the following selection criteria: initiated an infused biologic agent (abatacept, infliximab, or tocilizumab) between January 1, 2010 and January 1, 2012 (the dates of initiation for biologic agents used during this period were designated as the index dates); used at least one other biologic (either subcutaneous or an intravenous agent) at any time prior to the index date; had at least one inpatient or non-diagnostic outpatient medical claim (i.e., excluding medical claims such as radiology and venipuncture, which may represent services that are used to diagnose or rule out the presence of a condition) with a diagnosis of RA (ICD-9-CM code 714.0x) between January 1, 2009 and March 31, 2012; were aged 18 years or older on the index date; were continuously enrolled, according to insurance enrollment records, for at least 6 months pre-index (designated as the baseline period) and at least 3 months postindex; and had no medical claims with diagnosis codes for any non-RA indication of biologic agents (ankylosing spondylitis, chronic lymphocytic leukemia, Crohn's disease, juvenile idiopathic arthritis, polyarteritis nodosa, non-Hodgkin's lymphoma, plaque psoriasis, psoriatic arthritis, ulcerative colitis, or Wegener's granulomatosis) within the baseline period. Patients initiating treatments with induction doses were not necessarily required to complete the induction phase of treatment in order to be retained in the analysis.

An episode-based study design was used wherein patients were allowed to contribute multiple observations to the dataset, one for each infused biologic they initiated sequentially during the study period. Thus, patients were followed forward in time after their first qualifying infused biologic initiation to capture all subsequent episodes of infused biologic use. Episodes of infused biologic use began with initiation of a new infused biologic, with follow-up extending until the first occurrence of a 90-day gap in treatment with the initiated biologic, switch to a different biologic, insurance disenrollment, or the end of the study period (March 31, 2012).

Rituximab was not included in the analyses because of unique dosing intervals, which make it difficult to determine treatment discontinuation using a claims database. Specifically, the recommended frequency of rituximab re-infusions was initially based on clinical evaluation but later updated to be every 24 weeks or based on clinical evaluation, but not sooner than every 16 weeks. Because the magnitude of estimated healthcare costs incurred while a patient is being treated with a biologic is highly sensitive to the time period over which a patient is determined to be 'on treatment,' censoring follow-up at a 90-day gap may artificially inflate the cost of care for RA patients who are being treated with rituximab based on clinical evaluation.

\section{Healthcare Cost Outcomes}

The study outcomes were per-patient per-month (PPPM) healthcare costs measured during the follow-up. PPPM is calculated by dividing the total costs incurred during the episode by the number of days in the episode and then multiplying the resultant per-day costs by 30 to normalize costs to a 30-day (monthly) unit. The use of PPPM costs therefore accounts for the fact that patient follow-up is not necessarily equal for all patients. Healthcare costs were classified into (1) biologic costs (biologic drug + administration), (2) RA-related healthcare costs, which included costs for drugs used in symptomatic treatment [biologic drug + administration + non-biologic RA drugs (methotrexate, hydroxychloroquine, leflunomide, minocycline, sulfasalazine, cyclosporine, azathioprine, gold sodium thiomalate, non-steroidal anti-inflammatory drugs, corticosteroids, other analgesics) + medical claims coded with ICD-9-CM diagnosis codes for RA], and (3) all-cause healthcare costs (all pharmacy and medical claims). Healthcare costs were measured from the payment field on adjudicated medical and pharmacy insurance claims within the database. These costs include the gross covered payments for all healthcare services or products (i.e., the amount eligible for payment after applying pricing guidelines such as fee schedules and discounts, but including deductibles, copayments, and coordination of benefits). Healthcare costs were expressed in 2011 constant US dollars, adjusted using the Medical Care component of the Consumer Price Index [24]. 
Table 1 Patient demographics

\begin{tabular}{|c|c|c|c|}
\hline & $\operatorname{TCZ}(N=1,090)$ & $\mathrm{ABA}(N=1,759)$ & $\operatorname{INF}(N=922)$ \\
\hline Age (mean $\pm \mathrm{SD})$ & $54.7 \pm 12.3$ & $55.4 \pm 12.8$ & $53.5 \pm 13.2$ \\
\hline Female $(\%)$ & 83.1 & 83.2 & 81.2 \\
\hline \multicolumn{4}{|l|}{ Geographic region (\%) } \\
\hline Northeast & 12.3 & 11.8 & 13.3 \\
\hline North Central & 27.3 & 28.1 & 22.2 \\
\hline South & 39.0 & 42.0 & 43.5 \\
\hline West & 20.6 & 17.1 & 20.0 \\
\hline Unknown & 0.7 & 1.0 & 1.0 \\
\hline \multicolumn{4}{|l|}{ Insurance plan type $(\%)$} \\
\hline Comprehensive & 11.0 & 13.8 & 12.4 \\
\hline EPO & 1.7 & 1.9 & 1.3 \\
\hline HMO & 15.2 & 14.1 & 16.7 \\
\hline POS & 7.9 & 8.2 & 6.9 \\
\hline $\mathrm{PPO}$ & 53.9 & 53.2 & 55.0 \\
\hline POS with capitation & 0.9 & 0.9 & 0.8 \\
\hline CDHP & 3.7 & 2.9 & 2.4 \\
\hline HDHP & 1.7 & 1.8 & 1.6 \\
\hline Unknown & 4.0 & 3.2 & 2.9 \\
\hline \multicolumn{4}{|l|}{ Population density (\%) } \\
\hline Urban & 84.9 & 85.2 & 83.8 \\
\hline Rural & 14.4 & 13.8 & 15.2 \\
\hline Unknown & 0.7 & 1.0 & 1.0 \\
\hline \multicolumn{4}{|l|}{ Year of index $(\%)$} \\
\hline 2010 & 33.4 & 48.9 & 47.7 \\
\hline 2011 & 66.6 & 51.1 & 52.3 \\
\hline
\end{tabular}

$A B A$ abatacept, $C D H P$

Consumer Directed Health Plan, EPO Exclusive Provider

Organization, HDHP High

Deductible Health Plan, $H M O$

Health Maintenance

Organization, $I N F$ infliximab,

$P O S$ Point of Service, $P P O$

Preferred Provider

Organization, $S D$ standard

deviation, $T C Z$ tocilizumab

\section{Covariates}

The study covariates included patient demographics and clinical characteristics thought to potentially confound the relationship between the healthcare costs outcomes and biologic agent. Patient demographics were measured at index and are listed in Table 1. Patient clinical characteristics were measured throughout the baseline period and are listed in Table $2[25,26]$. The clinical characteristics included an administrative claims-based RA severity score [27]. This score has been shown to have moderate correlations with a previously validated recordsbased index of severity that had established construct and convergent validity with the Disease Activity Score in 28 Joints (DAS28). The Claims-based Index for Rheumatoid Arthritis Severity (CIRAS) is a numerical value that is computed on the basis of orders for inflammatory markers, number of platelet counts and chemistry panels ordered, rheumatoid factor, rehabilitation visits, age and gender, presence of Felty's syndrome, and number of rheumatology visits. Details on the algorithm can be found in Ting et al. [27]. The clinical characteristics also included two administrative claims-based indices of general health-the number of unique ICD-9-CM codes recorded on claims in the baseline period and the number of unique NDCs in the baseline period - that have been shown to correlate with healthcare costs and adverse outcomes such as emergency room visits and hospitalizations [25].

\section{Statistical Analyses}

Bivariate analyses were used to display summary statistics for the variable distributions, stratified by infused biologic agent. Multivariable generalized estimating equations (GEE) models-which accounted for the possibility of multiple observations per patient-were used to compare the healthcare cost outcomes between the infused biologic agents, adjusting for patient demographics and clinical characteristics listed in Tables 1 and 2 [28-30]. These models used an independent covariance structure, log link, and gamma distribution. Predicted adjusted incremental costs and their associated $95 \%$ confidence intervals were obtained via least squares means calculated in the framework of the GEE model relating costs to the covariates. All analyses were performed using SAS version 9.2 (Cary, NC, USA). $P$ values $<0.05$ were considered, a priori, to be statistically significant. $P$ values are reported only for 
Table 2 Patient baseline clinical characteristics

\begin{tabular}{|c|c|c|c|}
\hline & $\mathrm{TCZ}(N=1,090)$ & ABA $(N=1,759)$ & $\mathrm{INF}(N=922)$ \\
\hline Median follow-up in days & 251 & 280 & 269 \\
\hline CIRAS (mean \pm SD) & $3.6 \pm 0.9$ & $3.5 \pm 1.0$ & $3.6 \pm 1.1$ \\
\hline NSAIDs $(\%)$ & 38.5 & 39.3 & 41.9 \\
\hline Corticosteroids (\%) & 80.8 & 78.6 & 76.7 \\
\hline Analgesics (\%) & 63.9 & 58.6 & 56.9 \\
\hline Non-biologic DMARDs (mean \pm SD) & $0.9 \pm 0.7$ & $0.9 \pm 0.7$ & $1.0 \pm 0.7$ \\
\hline \multicolumn{4}{|l|}{ Non-biologic DMARDs (\%)* } \\
\hline Methotrexate & 50.3 & 50.9 & 61.2 \\
\hline Hydroxychloroquine & 13.3 & 16.4 & 16.6 \\
\hline Leflunomide & 14.1 & 13.3 & 15.1 \\
\hline Minocycline & 1.1 & 1.3 & 0.9 \\
\hline Sulfasalazine & 5.0 & 6.6 & 6.4 \\
\hline Cyclosporine & 0.2 & 0.2 & 0.3 \\
\hline Azathioprine & 5.4 & 3.2 & 2.2 \\
\hline Gold sodium thiomalate & 0.1 & 0.0 & 0.2 \\
\hline Extra-articular disease** $(\%)$ & 5.5 & 4.5 & 3.4 \\
\hline DCI $($ mean $\pm \mathrm{SD})$ & $1.4 \pm 0.9$ & $1.4 \pm 0.9$ & $1.4 \pm 0.9$ \\
\hline Number of unique 3-digit ICD-9-CM (mean \pm SD) & $21.7 \pm 16.6$ & $18.8 \pm 14.4$ & $18.7 \pm 14.6$ \\
\hline Number of unique NDCs (mean \pm SD) & $21.2 \pm 15.7$ & $17.8 \pm 12.3$ & $18.5 \pm 13.6$ \\
\hline Immediately prior drug $=$ anti-TNF $(\%)$ & 49.5 & 85.8 & 72.5 \\
\hline
\end{tabular}

$A B A$ abatacept, CIRAS Claims-based Index for Rheumatoid Arthritis Severity, DCI Deyo-Charlson Comorbidity Index, DMARD diseasemodifying antirheumatic drug, ICD-9-CM International Classification of Diseases, 9th Revision, Clinical Modification, INF infliximab, $N D C$ National Drug Code, NSAID non-steroidal anti-inflammatory drug, $S D$ standard deviation, $T C Z$ tocilizumab, TNF tumor necrosis factor- $\alpha$

* The overall number of non-biologic DMARDs was adjusted for in the models as opposed to the individual non-biologic DMARD indicators

** Rheumatoid nodules, Sjögren's syndrome, retinal vasculitis, other vasculitis, Felty's syndrome, or rheumatoid lung

multivariable-adjusted results, as these are the only analyses on which inferences were based.

\section{Results}

\section{Patient Characteristics}

Figure 1 displays the sample attrition associated with the application of each study inclusion and exclusion criterion. The final sample comprised 3,771 infused biologic initiations.

Tables 1 and 2 display patients' demographics and baseline clinical characteristics, respectively, stratified by infused biologic agent. The average patient age differed little across the biologics, ranging from 53.5 years in infliximab-treated patients to 55.4 years in abatacepttreated patients. The proportion of females also differed little across the biologics, ranging from $81.2 \%$ in infliximab-treated patients to $83.2 \%$ in abatacept-treated patients. The median follow-up ranged from 251 days among tocilizumab-treated patients to 280 days among abatacept-treated patients. Tocilizumab-treated patients had the numerically greatest proportions of patients with baseline use of corticosteroids, baseline use of analgesics, and presence of extra-articular disease, and the numerically greatest values of the number of unique 3-digit ICD-9-CM diagnosis codes and number of unique NDCs, all of which are indicative that tocilizumab-treated patients had poorer baseline health status than abatacept- and infliximabtreated patients.

\section{Healthcare Cost Outcomes}

Table 3 displays unadjusted PPPM biologic and RArelated healthcare utilization and costs. The mean PPPM [standard deviation (SD)] total RA-related healthcare costs were $\$ 2,815(\$ 3,615)$ for tocilizumab-treated patients, $\$ 2,936(\$ 2,038)$ for abatacept-treated patients, and $\$ 3,720$ $(\$ 3,713)$ for infliximab-treated patients. The majority of RA-related healthcare costs were driven by the cost of the biologic, accounting for $78.2 \%$ of the RA-related healthcare costs among tocilizumab-treated patients, $88.4 \%$ among abatacept-treated patients, and $87.2 \%$ among infliximab-treated patients. The mean (SD) count of biologic administrations was $1.0(0.2)$ for tocilizumab- 
Fig. 1 Sample selection attrition. $R A$ rheumatoid arthritis

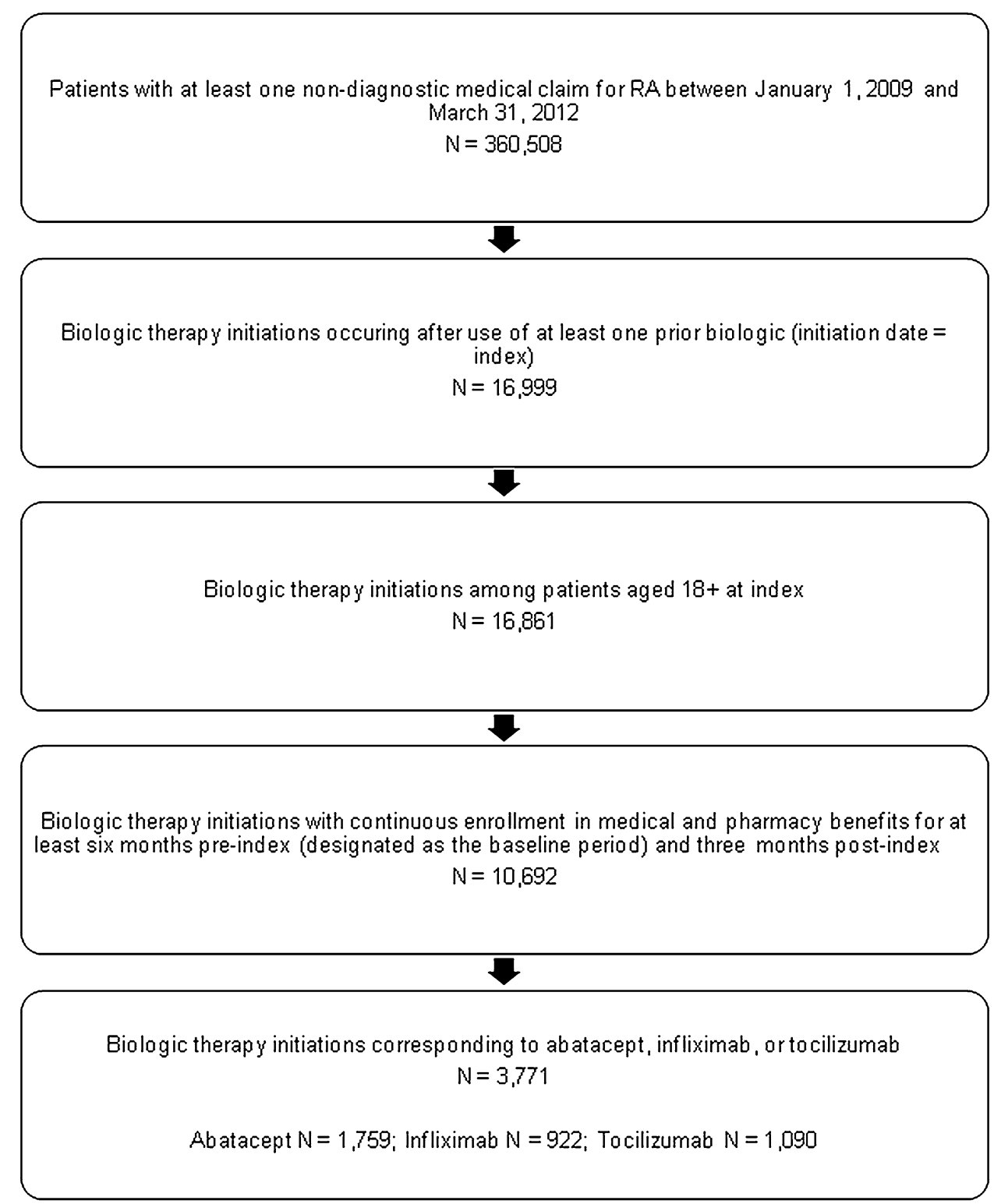

treated patients, $1.2(0.5)$ for abatacept-treated patients, and $1.0(0.5)$ for infliximab-treated patients.

Table 4 displays unadjusted PPPM all-cause healthcare utilization and costs. The mean PPPM (SD) total all-cause healthcare costs were $\$ 3,667(\$ 4,044)$ for tocilizumabtreated patients, $\$ 3,842(\$ 3,426)$ for abatacept-treated patients, and $\$ 4,739(\$ 6,058)$ for infliximab-treated patients. As with RA-related healthcare costs, the majority of allcause costs were driven by the cost of the biologic, which is captured in the 'other outpatient services' category.

Figure 2 displays multivariable-adjusted PPPM biologic, RA-related, and all-cause healthcare costs. When compared with abatacept-treated patients, tocilizumabtreated patients had mean (95\% confidence interval) multivariable-adjusted healthcare costs that were $\$ 703$
(608-798) lower for biologic costs, \$692 (550-834) lower for RA-related costs, and \$693 (485-900) lower for allcause costs (all $P<0.05$ ) (Table 5). When compared with infliximab-treated patients, tocilizumab-treated patients had mean (95\% confidence interval) multivariableadjusted healthcare costs that were $\$ 1,246(1,074-1,418)$ lower for biologic costs, \$1,362 $(1,123-1,601)$ lower for RA-related costs, and \$1,558 $(1,153-1,964)$ lower for allcause costs (all $P<0.05$ ).

\section{Discussion}

To our knowledge, this study is the first to compare healthcare costs between RA patients treated with infused 
Table 3 Unadjusted per-patient per-month biologic and RArelated healthcare utilization and costs
Healthcare costs were expressed in 2011 constant US dollars

$A B A$ abatacept, $I N F$ infliximab, $R A$ rheumatoid arthritis, $S D$ standard deviation, $T C Z$ tocilizumab

* Non-biologic DMARDs include methotrexate, hydroxychloroquine, leflunomide, minocycline, sulfasalazine, cyclosporine, azathioprine, and gold sodium thiomalate

** Other RA prescriptions include non-steroidal antiinflammatory drugs, corticosteroids, and other analgesics

\begin{tabular}{|c|c|c|c|c|c|c|}
\hline & \multicolumn{2}{|c|}{$\mathrm{TCZ}(N=1,090)$} & \multicolumn{2}{|c|}{$\mathrm{ABA}(N=1,759)$} & \multicolumn{2}{|c|}{$\mathrm{INF}(N=922)$} \\
\hline & Mean & SD & Mean & SD & Mean & SD \\
\hline \multicolumn{7}{|l|}{ Inpatient admissions } \\
\hline Count & 0.0 & \pm 0.1 & 0.0 & \pm 0.1 & 0.0 & \pm 0.1 \\
\hline Cost & $\$ 282$ & $\pm \$ 3,196$ & $\$ 106$ & $\pm \$ 893$ & $\$ 211$ & $\pm \$ 1,652$ \\
\hline \multicolumn{7}{|l|}{ Emergency room visits } \\
\hline Count & 0.0 & \pm 0.1 & 0.0 & \pm 0.1 & 0.0 & \pm 0.1 \\
\hline Cost & $\$ 6$ & $\pm \$ 54$ & $\$ 5$ & $\pm \$ 39$ & $\$ 4$ & $\pm \$ 37$ \\
\hline \multicolumn{7}{|l|}{ Outpatient office visits } \\
\hline Count & 0.7 & \pm 0.6 & 0.7 & \pm 0.6 & 0.7 & \pm 0.8 \\
\hline Cost & $\$ 67$ & $\pm \$ 88$ & $\$ 60$ & $\pm \$ 69$ & $\$ 63$ & $\pm \$ 69$ \\
\hline \multicolumn{7}{|l|}{ Other outpatient services } \\
\hline Count & 3.7 & \pm 3.3 & 3.3 & \pm 3.6 & 3.3 & \pm 3.7 \\
\hline Cost & $\$ 164$ & $\pm \$ 446$ & $\$ 109$ & $\pm \$ 317$ & $\$ 132$ & $\pm \$ 1,047$ \\
\hline \multicolumn{7}{|l|}{ Outpatient prescription claims } \\
\hline Count & 2.5 & \pm 1.2 & 2.7 & \pm 1.3 & 2.6 & \pm 1.7 \\
\hline Cost & $\$ 2,295$ & $\pm \$ 1,573$ & $\$ 2,657$ & $\pm \$ 1,749$ & $\$ 3,309$ & $\pm \$ 2,838$ \\
\hline \multicolumn{7}{|l|}{ Biologic DMARDs } \\
\hline Count of biologic administrations & 1.0 & \pm 0.2 & 1.2 & \pm 0.5 & 1.0 & \pm 0.5 \\
\hline Cost of biologic drug & $\$ 2,004$ & $\pm \$ 1,469$ & $\$ 2,356$ & $\pm \$ 1,693$ & $\$ 2,979$ & $\pm \$ 2,727$ \\
\hline Cost of biologic administration & $\$ 197$ & $\pm \$ 163$ & $\$ 240$ & $\pm \$ 190$ & $\$ 266$ & $\pm \$ 281$ \\
\hline \multicolumn{7}{|c|}{ Total cost of biologic drug + administration } \\
\hline Insurer-paid & $\$ 2,105$ & $\pm \$ 1,509$ & $\$ 2,469$ & $\pm \$ 1,685$ & $\$ 3,107$ & $\pm \$ 2,792$ \\
\hline Patient-paid & $\$ 97$ & $\pm \$ 152$ & $\$ 127$ & $\pm \$ 254$ & $\$ 138$ & $\pm \$ 317$ \\
\hline Total & $\$ 2,202$ & $\pm \$ 1,512$ & $\$ 2,596$ & $\pm \$ 1,745$ & $\$ 3,244$ & $\pm \$ 2,832$ \\
\hline \multicolumn{7}{|l|}{ Non-biologic DMARDs* } \\
\hline Count & 0.4 & \pm 0.5 & \pm 0.4 & \pm 0.5 & 0.5 & \pm 0.5 \\
\hline Cost & $\$ 16$ & $\pm \$ 39$ & $\pm \$ 13$ & $\pm \$ 29$ & $\$ 15$ & $\pm \$ 28$ \\
\hline \multicolumn{7}{|l|}{ Other RA Prescriptions** } \\
\hline Count & 1.1 & \pm 1.0 & \pm 1.0 & \pm 1.0 & 1.1 & \pm 1.4 \\
\hline Cost & $\$ 78$ & $\pm \$ 301$ & $\pm \$ 48$ & $\pm \$ 124$ & $\$ 50$ & $\pm \$ 165$ \\
\hline \multicolumn{7}{|c|}{ Total RA-related healthcare resource utilization } \\
\hline Insurer-paid & $\$ 2,668$ & $\pm \$ 3,547$ & $\$ 2,771$ & $\pm \$ 1,974$ & $\$ 3,542$ & $\pm \$ 3,667$ \\
\hline Patient-paid & $\$ 147$ & $\pm \$ 199$ & $\$ 165$ & $\pm \$ 269$ & $\$ 178$ & $\pm \$ 344$ \\
\hline Total & $\$ 2,815$ & $\pm \$ 3,615$ & $\$ 2,936$ & $\pm \$ 2,038$ & $\$ 3,720$ & $\pm \$ 3,713$ \\
\hline
\end{tabular}

biologics after previously using at least one other biologic agent. We found that among such patients, those treated with tocilizumab had the lowest real-world healthcare costs, largely driven by lower costs directly related to biologic treatment.

Owing to the uniqueness of the present study's data, there are very few studies to which ours can be compared. Wong et al. [20] used data from 72 US medical clinics to examine the drug and administration costs for abatacept, infliximab, and rituximab among RA patients initiating treatment between January 1, 2006 and December 31, 2008. They reported that mean (SD) unadjusted total costs per infusion visit were $\$ 1,827$ ( $\$ 622$ ) for abatacept, $\$ 2,828$ $(\$ 1,282)$ for infliximab, and $\$ 6,076(\$ 1,689)$ for rituximab.
Though the reported trend of infliximab being more costly than abatacept on a per-visit basis is consistent with the present study's findings on these two biologics, the study's cost comparisons were substantially confounded by the differences in the frequency of administration of the studied agents, which were unaccounted for in the per-visit cost comparisons. For example, abatacept is administered at 0 , 2 , and 4 weeks, and then every 4 weeks thereafter; infliximab, is administered at 0,2 , and 6 weeks, and then every 4-8 weeks thereafter; and rituximab is administered as two infusions separated by 2 weeks every 24 weeks or on the basis of clinical evaluation, but not sooner than every 16 weeks [5-7]. Thus, the data from Wong et al. [20] are difficult to interpret when trying to understand total drug 
Table 4 Unadjusted per-patient per-month all-cause healthcare utilization and costs
Healthcare costs were expressed in 2011 constant US dollars

$A B A$ abatacept, $I N F$ infliximab, $S D$ standard deviation, $T C Z$ tocilizumab

Fig. 2 Multivariable-adjusted (see Table 5) per-patient permonth healthcare costs. $A B A$ abatacept, $I N F$ infliximab, $R A$ rheumatoid arthritis, $T C Z$ tocilizumab

\begin{tabular}{|c|c|c|c|c|c|c|}
\hline & \multicolumn{2}{|c|}{$\operatorname{TCZ}(N=1,090)$} & \multicolumn{2}{|c|}{$\mathrm{ABA}(N=1,759)$} & \multicolumn{2}{|c|}{$\operatorname{INF}(N=922)$} \\
\hline & Mean & SD & Mean & SD & Mean & SD \\
\hline \multicolumn{7}{|c|}{ Inpatient admissions } \\
\hline Count & 0.0 & \pm 0.1 & 0.0 & \pm 0.1 & 0.0 & \pm 0.1 \\
\hline Cost & $\$ 415$ & $\pm \$ 3,353$ & $\$ 316$ & $\pm \$ 2,457$ & $\$ 505$ & $\pm \$ 4,414$ \\
\hline \multicolumn{7}{|c|}{ Emergency room visits } \\
\hline Count & 0.1 & \pm 0.2 & 0.1 & \pm 0.2 & 0.1 & \pm 0.2 \\
\hline Cost & $\$ 33$ & $\pm \$ 130$ & $\$ 41$ & $\pm \$ 265$ & $\$ 42$ & $\pm \$ 254$ \\
\hline \multicolumn{7}{|c|}{ Outpatient office visits } \\
\hline Count & 1.5 & \pm 1.0 & 1.4 & \pm 1.0 & 1.4 & \pm 1.2 \\
\hline Cost & $\$ 142$ & $\pm \$ 150$ & $\$ 126$ & $\pm \$ 109$ & $\$ 131$ & $\pm \$ 114$ \\
\hline \multicolumn{7}{|c|}{ Other outpatient services } \\
\hline Count & 9.2 & \pm 5.3 & 9.1 & \pm 6.0 & 9.6 & \pm 7.0 \\
\hline Cost & $\$ 2,701$ & $\pm \$ 1,762$ & $\$ 3,008$ & $\pm \$ 1,923$ & $\$ 3,727$ & $\pm \$ 3,927$ \\
\hline \multicolumn{7}{|c|}{ Outpatient prescription claims } \\
\hline Count & 4.3 & \pm 3.2 & 4.0 & \pm 3.1 & 4.1 & \pm 3.2 \\
\hline Cost & $\$ 376$ & $\pm \$ 593$ & $\$ 350$ & $\pm \$ 672$ & $\$ 334$ & $\pm \$ 688$ \\
\hline \multicolumn{7}{|c|}{ Total healthcare resource utilization } \\
\hline Insurer-paid & $\$ 3,428$ & $\pm \$ 3,959$ & $\$ 3,590$ & $\pm \$ 3,345$ & $\$ 4,468$ & $\pm \$ 6,009$ \\
\hline Patient-paid & $\$ 239$ & $\pm \$ 237$ & $\$ 252$ & $\pm \$ 306$ & $\$ 270$ & $\pm \$ 375$ \\
\hline Total & $\$ 3,667$ & $\pm \$ 4,044$ & $\$ 3,842$ & $\pm \$ 3,426$ & $\$ 4,739$ & $\pm \$ 6,058$ \\
\hline
\end{tabular}

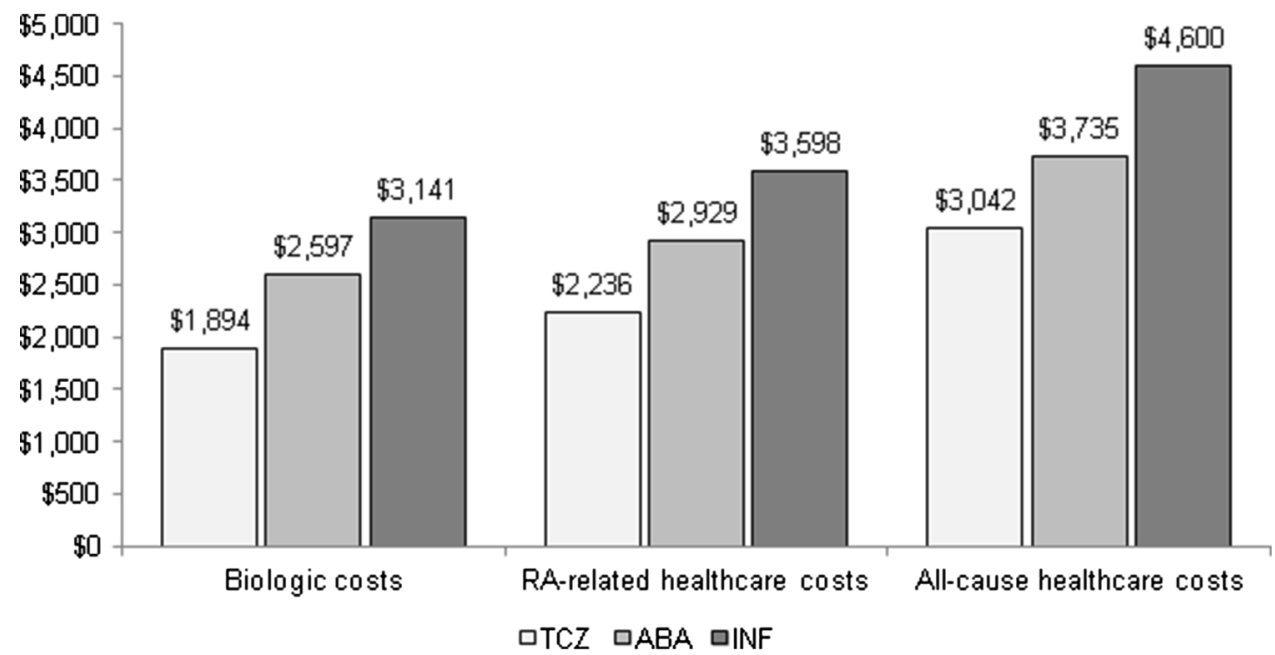

and administration costs that would be expected while a patient is being treated with infused agents.

In another prior study, Bonafede et al. [31] estimated annual biologic treatment costs for subcutaneous and infused biologics using a unit-cost approach, also finding differences among the infused biologic agents abatacept, infliximab, and rituximab. Results from the study were also somewhat consistent with the present analyses in that they, too, associated abatacept with lower average annual costs than infliximab ( $\$ 16,738$ vs. $\$ 21,273$, respectively). The analyses were intended to be purely descriptive, however, and made no attempts to adjust for inherent differences in patient characteristics that may drive differences in healthcare costs [21].

In one of the only other studies to report cost data for tocilizumab, Liu et al. [21] performed an indirect analysis of cost per responder in RA using clinical trial data and published drug acquisition and administration costs for FDA-approved RA biologics. They estimated that among the infused therapies, tocilizumab had the numerically lowest mean (95\% confidence interval) cost per responder at $\$ 31,363(14,713-64,232)$, compared with $\$ 50,496$ (25,819-92,069) for infliximab and \$61,088 (34,791104,295) for abatacept. Though these data are consistent 
Table 5 Multivariable-adjusted per-patient per-month cost differences

\begin{tabular}{lll}
\hline & \multicolumn{2}{l}{ Multivariable-adjusted* mean (95\% confidence interval) per-patient per-month cost difference** } \\
\cline { 2 - 3 } & ABA minus TCZ & INF minus TCZ \\
\hline Biologic costs & $\$ 703(608-798)$ & $\$ 1,246(1,074-1,418)$ \\
RA-related healthcare costs & $\$ 692(550-834)$ & $\$ 1,362(1,123-1,601)$ \\
All-cause healthcare costs & $\$ 693(485-900)$ & $\$ 1,558(1,153-1,964)$ \\
\hline
\end{tabular}

$A B A$ abatacept, $I N F$ infliximab, $R A$ rheumatoid arthritis, $T C Z$ tocilizumab

* The multivariable models adjusted all variables listed in Tables 1 and 2 (median follow-up excepted); adjusted costs are based on predictions set at the cohort-level mean values of continuous covariates and the base values of categorical covariates

** All cost differences were statistically significant at $P<0.05$

with the present study's findings regarding tocilizumab, it is important to consider that Liu et al.'s results were based on a simulation as opposed to being based upon actual observational data.

In the present study, the majority of all-cause costs were accounted for by the biologic therapies, suggesting that the real-world patterns of use of these therapies may be amongst the most important cost drivers for RA patients. The PPPM number of administrations for infliximab was 1.0 ; the expected number of administrations per month would be closer to 0.5 if infliximab were administered every 8 weeks as recommended for most patients on label. Thus, even after accounting for the more frequent induction doses $(0,2,6$ weeks), these higher than expected PPPM administrations suggest that dose escalation in the form of more frequent administration was occurring for infliximab. This is consistent with prior analyses that have suggested that dose escalation happens frequently in infliximab [11, 15, 17-19, 32]. While abatacept does not have a labeled option for dose escalation, tocilizumab may be prescribed at either $4 \mathrm{mg} / \mathrm{kg}$ every 4 weeks or $8 \mathrm{mg} / \mathrm{kg}$ every 4 weeks on the basis of clinical response $[5,8]$.

In a post hoc exploratory analysis of tocilizumab-treated patients, we attempted to estimate the proportion that dose escalated from $4 \mathrm{mg} / \mathrm{kg}$ to $8 \mathrm{mg} / \mathrm{kg}$ on the basis of changes in paid claim amounts. The Kaplan-Meier estimated probability of dose escalation from $4 \mathrm{mg} / \mathrm{kg}$ to $8 \mathrm{mg} / \mathrm{kg}$ within 1 year after initiation of tocilizumab was approximately $75 \%$. Given the relatively short follow-up of the present study (median ranging from 251 days in infliximab-treated patients to 280 days in abatacept-treated patients), further research should look at longer-term cost comparisons, which may be different from those reported in the present study, depending on the dosing patterns for each drug.

This study was subject to limitations. Procedure and diagnosis coding on administrative claims data are recorded by healthcare practitioners to support reimbursement. Measurement error can result from miscoded or non-coded administrative claims. For example, claims related to RA may not have been coded with an RA diagnosis code, thereby potentially underestimating the costs of RA. Though this study used multivariable analyses to adjust for differences in measurable patient characteristics between the infused biologics, we cannot rule out the possibility of residual confounding. Because of data limitations, we were unable to discern the exact line of therapy each patient was receiving. Compared with patients who are in earlier lines of biologic therapy, patients who have previously failed several biologics may be more refractory and may have higher RA-related costs, all-cause costs, and higher biologicrelated costs in part due to more dose escalation [32]. This study only compared the healthcare costs from a US payer perspective and did not compare the clinical effectiveness of the different treatments. Administrative claims data do not provide information on clinical outcomes such as tender/ swollen joint counts, pain, and physical function (among others). Thus, we could not assess the comparative clinical effectiveness of these therapies. Furthermore, the database does not have information on weight, which affects the total dose given to patients. However, using persistence on therapy as a proxy for effectiveness, our previous research has shown that tocilizumab patients had better persistence than patients receiving infliximab and abatacept [33]. Finally these results are not generalizable to the entire US RA population, including those who are uninsured or insured through Medicaid.

\section{Conclusion}

Among RA patients treated with infused biologics after previously using at least one other biologic, patients treated with tocilizumab had the lowest real-world healthcare costs, largely driven by lower costs directly related to biologic treatment. Such biologic-related cost differences may be driven by variations in real-world treatment patterns (e.g., dose, treatment frequency, dose escalation). 
Acknowledgments Support for this study was provided by Genentech, Inc. The authors wish to acknowledge the input/review of Jennie Best, $\mathrm{PhD}$, of Genentech. All named authors meet the International Committee of Medical Journal Editors criteria for authorship for this manuscript, take responsibility for the integrity of the work as a whole, and have given final approval to the version to be published.

Conflict of interest Stephen S. Johnston, Donna McMorrow, Amanda M. Farr, and Paul Juneau are employees of Truven Health Analytics, which was paid by Genentech, Inc. to conduct this study. Sarika Ogale is an employee of Genentech, Inc.

Compliance with ethics guidelines This article does not contain any new studies with human or animal subjects performed by any of the authors. The study databases satisfy the conditions set forth in Sections 164.514 (a)-(b)1ii of the Health Insurance Portability and Accountability Act of 1996 privacy rule regarding the determination and documentation of statistically de-identified data. Because this study used only de-identified patient records and does not involve the collection, use, or transmittal of individually identifiable data, Institutional Review Board approval to conduct this study was not necessary.

Open Access This article is distributed under the terms of the Creative Commons Attribution Noncommercial License which permits any noncommercial use, distribution, and reproduction in any medium, provided the original author(s) and the source are credited.

\section{References}

1. Singh JA, Furst DE, Bharat A, et al. 2012 update of the 2008 American College of Rheumatology recommendations for the use of disease-modifying antirheumatic drugs and biologic agents in the treatment of rheumatoid arthritis. Arthritis Care Res (Hoboken). 2012;64:625-39.

2. Williams EL, Edwards CJ. Patient preferences in choosing antiTNF therapies-R1. Rheumatology (Oxford). 2006;45(12):1575-6.

3. Chilton F, Collett RA. Treatment choices, preferences and decision-making by patients with rheumatoid arthritis. Musculoskeletal Care. 2008;6(1):1-14.

4. Zhang J, Xie F, Delzell E, et al. Trends in the use of biologic therapies among rheumatoid arthritis patients enrolled in the US Medicare program. Arthritis Care Res (Hoboken). 2013. doi:10. 1002/acr.22055 [Epub ahead of print].

5. Orencia [package insert]. Plansboro, NJ: Bristol-Myers Squibb; 2013.

6. Remicade [package insert]. Raritan, NJ: Janssen Biotech, Inc.; 2013.

7. Rituxan [package insert]. South San Francisco, CA: Genentech, Inc.; 2013.

8. Actemra [package insert]. South San Francisco, CA: Genentech, Inc.; 2013.

9. Deloitte Life Sciences. What payers want: viewing payers as customers. (2009). http://www.deloitte.com/assets/Dcom-United States/Local\%20Assets/Documents/us_lshc_WhatPayersWant 091109.pdf. Accessed 22 Aug 2013.

10. Managed Care. Payers step in with 'real-world' comparative effectiveness research. (2011). http://www.managedcaremag. com/archives/1106/1106.cer.html. Accessed 22 Aug 2013.

11. Harley CR, Frytak JR, Tandon N. Treatment compliance and dosage administration among rheumatoid arthritis patients receiving infliximab, etanercept, or methotrexate. Am J Manag Care. 2003;9(6 Suppl):S136-43.
12. Weycker D, Yu EB, Woolley JM, Oster G. Retrospective study of the costs of care during the first year of therapy with etanercept or infliximab among patients aged $\geq 65$ years with rheumatoid arthritis. Clin Ther. 2005;27(5):646-56.

13. Tang B, Rahman M, Waters HC, Callegari P. Treatment persistence with adalimumab, etanercept, or infliximab in combination with methotrexate and the effects on health care costs in patients with rheumatoid arthritis. Clin Ther. 2008;30(7):1375-84.

14. Carter CT, Changolkar AK, Scott McKenzie R. Adalimumab, etanercept, and infliximab utilization patterns and drug costs among rheumatoid arthritis patients. J Med Econ. 2012;15(2): 332-9. doi:10.3111/13696998.2011.649325 [Epub 2012 Jan 6].

15. Harrison DJ, Huang X, Globe D. Dosing patterns and costs of tumor necrosis factor inhibitor use for rheumatoid arthritis. Am J Health Syst Pharm. 2010;67(15):1281-7. doi:10.2146/ajhp090487.

16. Bonafede MM, Gandra SR, Watson C, Princic N, Fox KM. Cost per treated patient for etanercept, adalimumab, and infliximab across adult indications: a claims analysis. Adv Ther. 2012;29(3): 234-48. doi:10.1007/s12325-012-0007-y [Epub 2012 Mar 9].

17. Ollendorf DA, Klingman D, Hazard E, Ray S. Differences in annual medication costs and rates of dosage increase between tumor necrosis factor-antagonist therapies for rheumatoid arthritis in a managed care population. Clin Ther. 2009;31(4):825-35. doi:10.1016/j.clinthera.2009.04.002.

18. Ollendorf DA, Massarotti E, Birbara C, Burgess SM. Frequency, predictors, and economic impact of upward dose adjustment of infliximab in managed care patients with rheumatoid arthritis. J Manag Care Pharm. 2005;11(5):383-93.

19. Nair KV, Tang B, Van Den Bos J, et al. Categorization of infliximab dose changes and healthcare utilization and expenditures for patients with rheumatoid arthritis in commercially insured and Medicare-eligible populations. Curr Med Res Opin. 2009;25(2): 303-14. doi:10.1185/03007990802598736.

20. Wong BJ, Cifaldi MA, Roy S, Skonieczny DC, Stavrakas S. Analysis of drug and administrative costs allowed by US private and public third-party payers for 3 intravenous biologic agents for rheumatoid arthritis. J Manag Care Pharm. 2011;17(4):313-20.

21. Liu Y, Wu EQ, Bensimon AG, et al. Cost per responder associated with biologic therapies for Crohn's disease, psoriasis, and rheumatoid arthritis. Adv Ther. 2012;29(7):620-34. doi:10.1007/ s12325-012-0035-7 [Epub 2012 Jul 27].

22. Truven Health Analytics. MarketScan bibliography. http:// marketscan.truvenhealth.com/marketscanuniversity/publications/ 2012\%20Truven\%20Health\%20MarketScan\%20Bibliography.pdf Accessed 20 June 2013.

23. CPT copyright. [2009] American Medical Association. All rights reserved.

24. Consumer Price Index detailed report tables annual average. 2011. http://www.bls.gov/cpi/cpid12av.pdf. Accessed 15 March 2013.

25. Deyo RA, Cherkin DC, Ciol MA. Adapting a clinical comorbidity index for use with ICD-9-CM administrative databases. J Clin Epidemiol. 1992;45:613-9.

26. Fowler R, Johnston SS. (Podium presentation) Comparative performance of risk adjustment measures in a sample of commercially-insured patients under age 65-two simple measures outperform current standards. Value Health. 2010;13(3):A4.t.

27. Ting G, Schneeweiss S, Scranton R, et al. Development of a health care utilisation data-based index for rheumatoid arthritis severity: a preliminary study. Arthritis Res Ther. 2008;10:R95.

28. Cox DR. Regression models and life tables. J R Stat Soc B. 1972;34:187-220.

29. Huber PJ. The behavior of maximum likelihood estimates under nonstandard conditions. In: Proceedings of the fifth berkeley symposium on mathematical statistics and probability. 1967. Berkeley, CA: University of California Press, vol. 1, 221-233. 
30. White H. A heteroskedasticity-consistent covariance matrix estimator and a direct test for heteroskedasticity. Econometrica. 1980;48:817-30.

31. Bonafede M, Joseph GJ, Princic N, Harrison DJ. Annual acquisition and administration cost of biologic response modifiers per patient with rheumatoid arthritis, psoriasis, psoriatic arthritis, or ankylosing spondylitis. J Med Econ. 2013;16(9):1120-8. doi:10. 3111/13696998.2013.820192. [Epub 2013 Jul 18].

32. Ogale S, Hitraya E, Henk HJ. Patterns of biologic agent utilization among patients with rheumatoid arthritis: a retrospective cohort study. BMC Musculoskelet Disord. 2011;19(12):204. doi:10.1186/1471-2474-12-204.

33. Johnston S, McMorrow D, Farr A, Juneau P, Ogale S. Real world study of biologic disease modifying antirheumatic drug persistence among patients with rheumatoid arthritis who have previously used at least one other biologic agent: a US administrative claims database analysis. Abstract [2013] [SAT0566]. Presented at the European league against rheumatism 2013 Congress, Madrid, Spain, 12-15 June 2013. 